\title{
Anesthesia for Open Cholecystectomy in a Patient with Dilated Cardiomyopathy
}

\author{
Author
}

Dr Deepali Gomase Rahate

Sr. Consultant, Department of Anaesthesiology, Care Hospital, Nagpur

\begin{abstract}
Patients with limited reserve undergoing any noncardiac surgery is a challenge for anaesthesiologist and associated with high morbidity and mortality. Dilated cardiomyopathy is one of disorder with limited reserve. Anesthesiologist must be aware of different types of cardiomyopathies, associated preoperative evaluation and management of complications so as to conduct smooth anaesthesia. Patients with cardiomyopathy may be associated with other comorbidities and present to anesthesiologist for various emergency or elective cases. We reported a case of patient with dilated cardiomyopathy posted for open cholecystectomy. Along with DCM patient had history of chronic obstructive pulmonary disease with active rhonchi. We planned this case under thoracic epidural so as to avoid general anaesthesia.

Keywords: Anesthesia, thoracic epidural, dilated cardiomyopathy, Chronic obstructive airway disease (COPD).
\end{abstract}

\section{Introduction}

Dilated cardiomyopathy is disease of myocardium in which myocardial contractility reduces and biventricular or left ventricular function gets affected. It is the most common type of nonischemic cardiomyopathy and the third most common cause of heart failure. It is the commonest indication for cardiac transplantation also $^{(1)}$. It is defined by the presence of fractional myocardial shortening less than $25 \%$ and/or left ventricular ejection fraction (LVEF) less than $45 \%$; and LV end diastolic diameter greater than $117 \%$ excluding any known cause of myocardial disease $^{(2)}$. Known causes are ischaemic, valve dysfunction and post-viral infection. DCM can also be found in association with sickle cell disease, muscular dystrophy, excess alcohol, hypothyroidism, and some chemotherapy agents $^{(3)}$.

\section{Case Report}

A 75 yrs old female patient was posted for open cholecystectomy. Patient was a known case of dilated cardiomyopathy with EF 30\%, she was hypertensive with COPD, recently admitted and treated case of atrial fibrillation with fast ventricular rate and acute kidney injury from which she was recovering. On examination this geriatric patient was lying propped up on bed with respiratory rate $>30$, SPO2 on room air was 95$96 \%$, heart rate $87 / \mathrm{m}$, regular, Blood pressure was $130 / 70$, Bilateral rhonchi were present all over the chest. Her CBC was normal, KFT shows high creatinine 1.6, hyponatremia 128 , and potassium normal 3.9, LFT/PT/INR within normal limits. Her echo showed concentric LVH, Dilated RA/ $\mathrm{RV}$, severe LV systolic dysfunction, with EF 30 $\%$. In view of bilateral rhonchi we decided to wait a day or two as surgeon thinks it is not an 
emergency. Patient was put on bronchodilators, nebulisation started but the condition remains same even after two days. High-risk consent was taken from the patient and relatives and regional anesthesia technique explained. After intravenous line secured with $18 \mathrm{~g}$ vasofix, left radial artery cannulated with $20 \mathrm{~g}$ and right internal jugular vein cannulation done with triple lumen under USG guidance under local anesthesia. Emergency drugs were kept ready, along with dopamine and noradrenalin infusion, lignocaine and amiadarone. Nebulisation and deriphylline continued preoperatively along with ondansetron and pantocid. The epidural space was identified by the "loss of resistance" technique in the T9-10 intervertebral space. An 18-gauge epidural catheter was inserted and advanced $5 \mathrm{~cm}$ cephalad. A test dose of $3 \mathrm{~mL}$ of $2 \%$ lidocaine with adrenaline (1:200000) was administered, then $5 \mathrm{~mL}$ of $0.5 \%$ bupivacaine was given after excluding intravenous or intrathecal spread. Two $\mathrm{ml}$ of bupivacaine repeated after 10 minutes. The height of block was tested by pin prick every 5 minutes. The surgery was started after confirmation of a block extension from T4 to T10 dermatomes. As patient was too anxious so she was sedated her with $1 \mathrm{mg}$ midazolam and $50 \mathrm{mcg}$ fentanyl. Small dose of dopamine started through central line @ 2-4 ml/hr so as to maintain blood pressure between 120-130mg. Procedure took 45 minutes, patients vitals remained stable throughout the procedure. $\mathrm{O}_{2}$ continued by mask at 4litres/minute. Postoperatively sensorcaine $0.125 \% @ 5 \mathrm{ml} / \mathrm{hr}$ continued through epidural for 48 hrs. Patient was discharged in stable condition on $5^{\text {th }}$ day.

\section{Discussion}

Laparoscopic cholecystectomy is the most widely performed laparoscopic procedure which requires gas i.e. carbon dioxide insufflation. Carbon dioxide considered as an ideal insufflating gas in laparoscopy as it is rapidly absorbed. But pneumoperitoneum in an anaesthestised patient in reverse tredelenberg position causes many hemodynamic changes in the form of increase in blood pressure, tachycardia, arrhythmia and even cardiac arrest have been reported. As our patient had active rhonchi so decided to go for regional anaesthesia and open cholecystectomy. Goals for anesthetic management in patients with dilated cardiomyopathy consist of maintaining normovolemia, prevention of increase in afterload and avoidance of drug induced myocardial depression. avoid tachycardia; control arrhythmias and to maintain adequate preload in the presence of elevated $\operatorname{LVEDP}^{(3,4)}$. Regional anaesthesia reduces the afterload which improves cardiac output, it also provides postoperative pain relief and helps to maintain better hemodynamics ${ }^{(5,6)}$. Some anesthesiologist reported a successful use of combined spinal epidural for non cardiac surgery in a patient of $\operatorname{DCM}(1,7)$. We use titrated epidural so as to easily control hypotension and to prevent myocardial hypoperfusion. If inotropic support requires during and after surgery, it can be provided by the use of a variety of agents, including dobutamine, dopamine, phosphodiesterase inhibitors, and levosimendin. ${ }^{(4)}$, norepinephrine can be used cautiously to counteract some of the peripheral vasodilator effects of general anaesthetic agents to assist coronary perfusion. Many anesthestist reported the use of regional anaesthesia alone ${ }^{(8,9)}$ or in combination of total intravenous anaesthesia (10)or with general anaesthesia in a patient of dilated cardiomyopathy posted for different non cardiac surgeries .Perioperative risk such as arrhythmias and cardiac failure should be kept in mind and drugs like xylocard,, metoprolol, amiadarone should be kept ready. Pulmonary and systemic embolism can occur as blood stasis in dilated and hypocontractile cardiac chambers and this lead to activation of the coagulation cascade $^{(11)}$. Invasive blood pressure monitoring done so as to detect early hypotension and CVP to roughly optimize the fluid requirement and for continuing inotropic support if required. Epidural anaesthesia is a reasonably safe and in our case we required a low dose of dopamine support so as to 
maintain blood pressure. Epidural also provides good postoperative pain relief and better hemodynamic stability and prevents vomiting too. It has been noticed that there is no apparent difference in the length of hospital stay and patient's satisfaction level increases with regional anaesthesia as compared to general anaesthesia, because of better quality of pain relief and absence of postoperative nausea and vomiting. Regional anesthesia proved more economical than GA in most of the cases ${ }^{(12)}$. Sometimes there may be asymptomatic non-sustained ventricular tachycardia and Arrhythmias which may be because of low levels of potassium and magnesium levels These electrolytes should be assessed preoperatively and corrected as necessary (7).and is managed with antiarrhythmics, for example, amiodarone or AICD device which improves survival $(13,14)$. AICD is a artificially implantable cardioverter defibrillator and detects arrhythmias and acts as a defibrillator and prevents sudden cardiac death due to sustained ventricular tachycardia or fibrillation. Cardiac transplantation significantly improves the life expectancy of patients with dilated heart failure but because of limited donor supply as well as lack of facilities for transplantation makes it rare choice for the patient.

\section{Conclusion}

Patients with cardiomyopathy carries significant risk of cardiac failure intraoperatively as well as postoperatively and should be assessed meticulously preoperatively. If it is associated with other diseases like COPD then it adds more careful and planned management. Proper preoperative evaluation, titrated doses of anaesthesia drugs, planning for emergency complications and proper monitoring can lead to favorable outcome.

\section{Conflict of interest: Nil}

\section{References}

1. Ravi Raj, Mritunjay Kumar, and Meenu Batra. Anesthetic management of a case of dilated cardiomyopathy for emergency appendectomy, Anesth Essays Res. 2014 Jan-Apr; 8(1): 105-107

2. Wood WL, Kuczkowski KM, Beal BR. Anesthetic considerations for cesarean section in the parturient with familial cardiomyopathy. Acta Anaesthesiol Belg. 2008;59:87-9. [PubMed] [Google Scholar])

3. M.R. Davies, J. Cousins, Cardiomyopathy and anaesthesia Continuing Education in Anaesthesia Critical Care \& Pain, Volume 9, Issue 6, December 2009, Pages 189-193,

4. Rajiv Juneja, Prajeesh M , Cardiomyopathies and anaesthesia, IJA REVIEW ARTICLE Year : 2017 | Volume : 61 | Issue : 9 | Page : 728-735

5. Narang S. Adequate pain management contributes to hemodynamic stability. Middle East J Anaesthesiol 2007;19:243-9.

6. De Hert SG, Van der Linden PJ, ten Broecke PW, Vermeylen KT, Rodrigus IE, Stockman BA, et al. Effects of desflurane and sevoflurane on length-dependent regulation of myocardial function in coronary surgery patients. Anesthesiology 2001;95:357-63.

7. van Zundert AAJ, Stultiens G, Jakimowicz JJ, et al. Segmental spinal anaesthesia for laparoscopic cholecystectomy in a patient with severe lung disease. Brit $\mathrm{J}$ Anaesth 2006; 96:464-6.

8. R. Barani Selvan, David George Veliath, Parnandi Bhaskar Rao, Ramachandran, and R. V. Ranjan, Cholecystectomy under segmental thoracic epidural block in a patient with twin gestation, Saudi J Anaesth. 2012 Jan-Mar; 6(1):73-75

9. Gramatica L, Brasesco OE, Mercado Luna A, et al. Laparoscopic cholecystectomy performed under regional anesthesia in patients with chronic obstructive pulmonary disease. Surg Endosc 2002; 16:472-5. 
10. Yamaguchi S, Wake K, Mishio M, Okuda Y, Kitajima T. Anesthetic management of a patient with dilated cardiomyopathy under total intravenous anesthesia with propofol and ketamine combined with continuous epidural analgesia. Masui. 1999;48:1232-4. [PubMed]

11. Davies MR, Cousins J. Cardiomyopathy and anaesthesia. Continuing Education in Anaesthesia, Critical Care \& Pain 2009;9(6) 189-93. Back to cited text no. 7

12. Muhammad Umar Zahoor1, Rehan Masroor2, Tassadaq Khurshid3, Raheel Azhar3and Malik Muhammad Amjad Yasin1Thoracic Epidural Anaesthesia for Open Cholecystectomy, ,Journal of the College of Physicians and Surgeons Pakistan 2011, Vol. 21 (11): 654-658

13. Oliver WC, Mauermann WJ, Nuttal G. Uncommon cardiac diseases. In: Kaplan J, Reich DL, Savino JS, editors. Kaplan's Cardiac Anesthesia. 6th ed., Ch. 22. Philadelphia: Elsevier Saunders; 2011. p. 675-736.

14. Desai AS, Fang JC, Maisel WH, Baughman KL. Implantable defibrillators for the prevention of mortality in patients with nonischemic cardiomyopathy: A meta-analysis of randomized controlled trials. JAMA 2004;292:2874-9. 